\title{
Organische Chemie.
}

1.

Microscopische Untersuchungen über die Hefe und Essignutter, nebst mehreren andern dazu gehörigen vegetabilischen Gebilden.

Von

Friedrich K̈̈тziNG,

Lehrer der Naturwissenschaften an der Realsthule zu Nordhausen.

(Im Auszuge vorgetragen in der Yersammlung des naturbistorischen Vereins des Marzes, in Alexisbad am 26. Juli 1837.)

(Mit Abbildungen auf tab. II. u. III.)

Als ich mich vor drei Jahren mit den microscopischen Untersuchungen der niedern, in verschiedenartigen Flüssigkeiten sich bildenden, vegetabilischen Organismen beschäftigte, untersuchte ich anch die Hefe und Essigmutter, Producte, welche sich bekanntlich bei der weinigen und sauren Gährung bilden. Schon damals war ich überzengt, dass die Hefe eine rein vegetabilische Bildung sei, und dieselbe nicht gleiche Bedeutung mit mehreren andern, sogenannten chemisch-organischen Verbindungen habe, wie man bisher in der Chemie die Hefe betrachtete.

Die Untersuchungen über diesen Gegenstand theilte ich nebst einigen andern physiologischen Untersuchungen schon im Herbst 1834 den Herren Professoren Horkel und Ehrenberg in Berlin mit und erwähnte sie anch beiläufig im mündlichen Gesprïche gegen Herrn Alexander von Humboldt.

Eine Reise nach Dalmatien, Italien und der Schweiz, die ich liurz nach jener Zeit unternahm, war die Ursache, dass Journ. f. prakt. Chewie. XI. 7. 
meine Untersuchungen nicht bekannt gemacht wurden, denn ich beabsichtigte diese Entdeckungen dem physiologischen Theile eines algologischen Werkes, welches ich za bearbeiten mir vorgenommen habe, cinzuverleiben.

Seit meiner Rückkehr von der Reise mehrten sich bei mir die Untersuchungen über diesen Gegenstand; und eben batte ich begonnen die Resultate darüber zusammenzustellen, als ich von meinem Freunde, dem Herrn Hofrath Walle oth auf dieselbe Entdecknng des Herrn Cagniard-Latour in Betreff der Hefe anfmerksam gemacht wurde. Kurz darauf (im September d. J.) erbielt ich dio Annalen der Physik und Chemie, und sah die Abhandlung des Herrn Sehwann in Berlin, welcher äbnliche Resultate in Betreff der Untersuchungen über die Hefe erhalten batte.

Indem nun von uns Dreien ein und dasselbe in Hinsicht auf die wirklich organische *) Natur der Hefe beobachtet wurde, obne dass einer von den Untersuchungen des andern Kunde batte, so ist mir dies um so erfreulicher, da ich meine Beobachtungen auch durch andere Naturforscher bestätigt sehe. Ich verzichte darum gern auf das Prioritütsrecht einer Entdeckung, da es für die Wissenschaft doch gleich ist, wer es zuerst fand, und ich zudem überzeugt bin, dass die Mlittheilung meiner Beobachtungen dennoch nicht überflüssig sein dürfte, indem sie noch einige Thatsachen enthalten, die in den Mittheilangen der Herrea Cagniard-Latour und Schwann nicht enthaiten sind.

Uebrigens muss ich hierbei bemerken, dass schon Desmazières vor 10 Jahren die Hele nebst ihren Metamorphosen beobachtet und in den Aunales des sciences naturelles l'om. X. p. 42-67. unter dem Namen Mycoderma cerevisiae, nebst noch einigen andern hierher gehörigen Arten beschrieben und abgebildet bat. Herrn Desmazières würde daher das Prioritätsrecht dieser Entdeckung zukommen, wenn er sein Mycoderma cerevisiae sogleich für die Hefe erkannt, und nicht unglücklicher Weise für ,animalcula monadina" gehalten hätte.

$Z \mathbf{Z}$ jener Zeit, als ich mich von der vegetabilischen Nalur

*) Wirklich organisch nenne ich sie desshalb, um sie von den fitschlich sogenannten chemisch-organischen Verbindungen, wic z. B. Zucker, Alkohol, Aether etc. zu unterscheiden. 
der Hefe überzeugt batte, fand ich auch, dass der schleimige Absatz, der sich nach einiger Zeit stets in der Tinctura rhei aquosa der Apotheker bildet, ebenfalls vegetabilischer Natur sei *). Eben so fand ich, dass auch die Essignutter, die sich beim Gähren des Essigs auf der Obcrntiche bildet, denselben Gebilden zuzuzählen sei, wie die Ausgabe der 11ten Decade meiner Algae aquase dulcis etc. bezeugt, in welcher ich die Essigmutter unter dem Namen Ulvina aceti geliefert habe.

Dass sich auf äbnliche Weise in verschicdenen Solutionen chemischer Präparate vegetabilische Organismen bilden, ist den Algologen durch die Werle Agardh's 米籼 und Biasoletto's 类类) bekannt, welche eine Anzahl solcher niedern Pflanzenformen beschrieben haben. Auch von mir wurden einige dieser niedern vegetabilischen Organismen in meinen Decaden der Süsswasseralgen geliefert.

Ohne mich noch weiter über die geschichtlichen Nachweisungen solcher Untersuchungen auszudehnen, werde ich nun eine Reihe von Beobachtungen folgen lassen, die ich seit mebreren Jabren gesammelt habe.

\section{Die Hefe. Fig. I. his V.}

Betrachten wir die Hefe, welche sich beim Gühren des Bieres und der Maische bildet, unter dem Microscope, so erblicken wir eine Menge sehr kleiner kugeliger oder ovaler Kügelchen (Fig. I.), welche ganz farblos und durchscheinend sind. Die ovale Gestalt tritt besonders bei starker, ohngefïhr 420maliger Linear - Vergrösserung 米粎楼) deutlich hervor. Diese Kügelchen sind nicht immer von gleicher Grösse; im Durchschnitt beträgt ihr Durchmesser $\frac{1}{3} v^{\prime \prime \prime}$, bei den grössern $\frac{1}{251} " \prime$, bei den kleinern $\frac{1}{3} \sigma^{\prime \prime \prime}$. Bei etwas matter Beleuchtung tritt in dem Centrum dieser Kügelchen ein etwas opaker runder Kern hervor, der durch eine kreisförmige Linie begrenzt wird, und oft scheint sich ein zweiter Kreis um den ersten zu zieben, wodurch die Structur dieser Kügelchen ein concen-

*) Vergl. Journal für praktische Chemie. 1834. I. p. 475.

**) Species algarum p. 45-49.

来为) Di alcune alghe microscopiche etc.

*3*;:) Alle diese inicroscopischen Untersuchungen sind mit einem Microscope aus der Werlistatt des Herin Schiek in Berlin gemacht. 
388 Kützing, üb. Hefe und Essigmutter.

trisches Ansehen bekommt, ähnlich der Structor der Stärkekügelchen, von welcben sie sich beim ersten Anblick auch wirklich nur durch bedeutend grössere Kleinheit und durch die geringere Anzahl der concentrischen hinge unterscheiden. Ich kochte die Hefeliügelchen mit Wasser, konnte dadurch aber keine bemerkbare Veränderung derselben bewirken; jedoch wurde das Wasser etwas weniges schleimig. Dasselbe war auch der Fall, als der kochenden Flüssigkeit einige Tropfen Sehwefelsäure hinzugefügt wurden; nur erst dana, als noch mehr concentrirte Schwefelsäure hinzukam, quoll die Flüssigkeit zu einer schleimigen Masse, die zuletzt gallertartig wurde. Hinzugefügtes Wasser löste die gallertartige Masse selbst durch Kochen nicht aup, sondern letztere schwamm flockenartig darin umher. Unter dem Microscope erschienen die flockigen Schleimmassen als Aggregate von Hefekügelchen, welche zum Theil an Grösse verloren hatten, doch ohne das Eigenthümliche ihrer Structur verloren zu haben. Es schien als wenn nur die äusserste Schicht derselben eine Auflösung durch die Schwefelsäure erfahren hütte und dadurch in Gallert umgewandelt worden wäre. Darch diesen Schleim hingen die Kügelchen noch mit einander zusammen. Von manchen Kügelchen hatte sich mehr Substan\% aufgelöst als von andern, so dass von ihnen nur noch der innere Kern übrig geblieben war. Ein Zerplatzen der äussern Haut, wie es bei ähnlicher Belandlung mit dem Stärkmehl der Fall ist, wobei die Hänte ihres Inhaltes beraubt werden und dann in der Flüssigkeit umher schweben, kommt bei der Hefe nicht vor.

Dieses eigenthümlicbe Verhalten der Iefekügelchen, welches von dem der Stärke völlig abweicht, veranlasst mich zu der Annahme, dass die Hefekügelchen aus übereinander gelagerten Schichten bestehen, die aber sïmmtlich von gleichfester Substanz gebildet sind, so dass die innere Substanz eben so dauerhaft erscheint als die äassere.

Hiervon scheinen die Beobachtungen des Merrn Cag niardLatour, die ich jedoch nur aus den Nachsatze zu Herrn Schwanns Abhandlung kenne, abzuweichen, da derselbe das Ausströmen von Flüssigkeit aus den Hefekügelchen beobachtet haben will, welches aber nach meinen wiederholten Beobachtungen nicht stattfindet und auch nicht stattinden kann, da der 
innere Kern nicht flüssig, sondern solide ist. Während dieser Untersuchungen erhielt ich aus einer Brandtweinbrennerei eine andere hefenartige Masse, die sich aussen an einem Maischgefässe angesetzt hatte. Sie war schon sauer geworden und unter dem Microscope zeigten sich ausser den mir schon bekannten Hefekügelchen die beliannten Essigälchen. Ich liess sie, mit etwas Wasser verdünnt, in einem Schülchen längere Zeit stehen und bemerkte nach und nach folgende Veränderung daran:

Nach einigen Tagen bildeten sich auf der Oberfäche kleine, zarte Hüutchen, welche aus Hefekügelchen bestanden, die sich auf die Oberlïche begeben hatten; ich bemerkte zugleich nnter dem Microscope, dass mehrere Kügelchen aneinander gereiht, und selbst zu einem kurzen Faden verwachsen waren (Fig. II.). Späterhin wurde das Verwachsen der Kügelchen aur der Oberlïche noch deutlicher, so dass man sogar ästige, gegliederte Fäden unterscheiden konnte. Die Glieder waren bei einigen Füden nicht verschieden von den Hefekügelchen, bei den meisten aber hatten sie sich mebr verlïngert. In solchen verlängerten Gliedlern bemerkte ich auch mehrere opake runde Punkte, meist zwei oder drei.

Hie und da waren noch einige, verlängerte Glieder zu bemerken, welche fast die Gestalt cines Oblong hatten, theils einzeln herum lagen, theils zu einem Faden verbunden waren. Diese Veründerung war jedoch nicht an denjenigen Itelekügelchen vorgegangen, welche noch innerhalb der feuchten Masse befindich waren, sondern nur an den auf der Oberläche befindichen.

In einer andern schale batte sich die Hefenmasse auf ihrer Oberfliche fast ganz in jene erwähnten oblongartigen Körperchen (Fig. III.) umgewandelt, und zeigte jene gegliederten und ästigen Fäden in weit geringerer Anzahl. Diese länglichen Körperchen waren theils quer, theils der Lïnge nach ancinander gereiht und durch das Vertheilen dieser Massen im Wasser trennten sie sich oft in der Weise, dass dadurch tihnliche zickzackartige, oder auch wohl strahlige Figuren entstanden, wie wir sie unter den Djatnmeen bei den Gattungen Diatoma und Exilaria gewahr werden. Fin anderer Theil dieser oblongartigen Körperchen war jedoch auch schon zu Fä- 
den ausgewachsen, die undeutlich und ungleich gegliedert erschienen.

Obgleich nach und nach die Hefe eintrocknete, vegetirte dennoch die Oberfäche immer fort, die Fïden wurden länger, zugleich aber auch dünner, eben so erzeugten sicb auch noch neve Kügelchen, diese waren aber kleiner, wie die Prühern Hefekügelchen. Die ganze Masse bekam nun ein schimmelartiges Ansehen; nach kurzer Zeit wurden die kleinern, neugebildeten Kügelchen bläulichgrau gefärbt und es batte sich ein Sporotrichum gebildet (Fig. IV.).

An einer andern Stelle der Schale, die etwas feuchter geblieben war, wurden die Fäden länger und dicker, sie zeigten hie und da Anschwellungen der Glieder, am meisten aber schwollen die Endglieder an, sie bildeten kngelige Köpfchen und bald batte sich ein völliger Mucor ausgebildet (Fig. V.).

\section{Die Essigmutter (Fig. VI. bis VIII.).}

Essigmutter wird diejenige schleimige and gelatinose Masse genannt, welche sich bei der sauren Gährung auf der Oberfläohe der gährenden Flüssigkeit zeigt. Ich babe die Essig mutter von verschiedenartigen, der sauren Gübrung unterworfenen Flüssigkeiten untersucht, und sie stets in ibrer Bildung gleichartig gefunden.

Die Bildung der Essigmutter beginnt sogleich mit der Essigbildung und schreitet mit dieser in gleichem Verhältnisse fort, Der erste Anfang (Fig. VI.) besteht in einem dünnen bäutigen Anfluge, welcher die Oberfläche der gährenden Flüssigkeit überzieht. Dieses Häutchen hat jedoch nur noch sehr geringen Zusammenbang. Unter dem Mieroscope sieht man, dass es ans ausserordentlich kleinen Kügelchen besteht, die wohl noch um 6mal kleiner als die Hefekügelchen sind und einen Durchmesser von $\frac{1}{2000}$ bis $\frac{1}{150 \pi}$ "' baben. Zuweilen schien es mir, als wären diese Kügelchen in bestimmter Ord̉nung reiluenweise aneinander gelagert; meist waren jedoch die Kügelchen obne bestimmte Ordnung aneinander gefügt, wurden von einem umhüllenden Schleime zusammengehalten und waren nicht eigentlich zusammengewachsen. Wegen der ausserordentlicben Kleinheit der Kügelchen habe ich Nichts weiter an ihnen bemerken können, wonach man auf ihre Structur noch hätte schliesson 
können. Die weitere Ausbildung dieser Haut besteht darin, dass sie dicker, compacter und zusammenbüngender wird (Fig. VII.). Gewöhnlich babe ich gefunden, Jass sich dann (ohngetähr in einer Zeit von 14 Tagen) anch die äussere Gestalt bestimmter entwickeit. Denn sucht man eine ausgebildete dicke Haut von Essigmutter in kleinere 'Theile zu trennen, sa bemerkt man, dass die ganze Haut aus dicht nebeneinander liegenden, vertikalen, and zuweilen diclıotomisch verïstelten Frondes besteht, die an die ähnliche Biluung einiger Meeralgen z. B. der Halymenia furcata erinnern.

Eine so ausgebildete Essigmutter gewährt daher gleichsam ein gelatinos-fucoidisches Ansehen, wie die Frons einer asstigen Chaetophora. Die festere Beschaffenheit der Essigmutter in diesem stadium rührt daher, dass die innern Kügelchen durch den umhüllenden Schleim inniger mit einander verwachsen. Da aber hierdurch die Kügelchen mit ihrer schleimigen Umgebung inniger verschmelzen, so siebt man sie in dieser Entwicklungsperiode weniger deutlich als in der ersten, denn ihr Umriss ist weniger scharf begrenzt.

Einige Hänte von ausgebildeter Essigmutter, die ich noch mehrere Tage in Wasser aufbewahrte, veränderten sich in ihrem Intuern dergestalt, dass es nicht mehr aus. blossen kleinen Kügelcben, sondern aus länglichen Körperchen bestand (Fig. VIII.), die durch das Verwachsen der Kügelchen entstanden zu sein schienen, wenigstens konnte ich bei vielen eíne deutliche Gliederung benerken, dic durch jenes Verwachsen veranlasst worden war.

Diese Thatsachen waren mir za interessant als dass ich sie nicht mit mehreren andern und älınlichen hätte in Vergleich bringen sollen. Dies glaubte ich uth so mehr thun zu müssen, da sie wichtige Aufschlüsse nicht nur übor die Entsteliung Jer Pilze und niedern Algen, als auch über die generatio primitiva geben können.

Ich habe seit einer Reihe von Jahren eine grosse Anzahl niederer vegetabilischer Organismen beobachtet und jedesmal ähnliche Verhältnisse hinsichtlich ihrer Entstehung und fernem Entwicklung bemorkt. Dass sich die niedern Vegetabilien, so- 
wohl niedere Algen als Schwämme durch Urbildung erzeugen, daran zweifele ich' jetzt keinen Augenblick mehr, dass es aber von Umständen, dem geringern oder grössern Feachtigkeitsoder Wärmegrade, der An - oder Abwesenheit von Licht etc. abhängt, welche Form sich gerade ausbilden soll, dies habe ich schon in einer frühern Abhandlung , über die Lintstebung und Metamorphose der niedern vegetabilischen Organismen " in der Linnaea 1833. Heft III. ausgesprochen und wiederhole es jetzt mit um so grŏsserer Ueberzengung.

Eben so übt die chemische Beschaffenheit der Flüssigkciten, in welchen sich diese niedern Organismen bilden, Einfluss auf die Form der Bildungen. Den Beleg hierzu liefern die Thatsachen, welche ich weiter unten mittheile. Für die Physiologie werden hierdurch, wie ich hoffe, wichtige Aufschlüsse hervorgehen, und auf der andern Seite dürften sie für die fernere Bearbeitung der sogenannten organischen Chomie den grōssten Einfluss ausüben. Hier ist es, wo das Studium dieser niedern Geschöpre für das ganze naturwissenschaftiche Feld von ausserordentlicher Wichtigkeit werden kann, während die blosse Betrachtung der einzelnen, sehr mannichfaltigen Formen nach and nach mehr in den Hintergrund treten muss.

Es versteht sich von selbst, dass jetzt die Chemie die Hefe unter der Zahl ibrer chemischen Verbindungen zu streichen habe, da sie keine chemische Verbindang, soudern ein organischer Körper, ein Organismus ist. Leider werden immer norh za viele wahrhaft organisirte Bildungen unter den chemischen Verbindungen angeführt, wo sie gar nicht hingehüren. Diese und viele andere Thatsachen, welche uns bei physiologischen Untersuchungen aufstossen, sind es aber auch, die es jetzt mehr als jemals wünschenswerth machen, dass sich hier die Wissensenschaften vereinigen, um Begrife festrustellen, die jetzt in den verschiedenen $Z$ weigen der Naturwissenschaften auch einen verschiedenen Sinn baben.

Ich erinnere hier nur daran, wie man den Begriff organisch in der Chemie und in der Naturgeschichte nimmt. Man hat sich hierüher noch nicht vereinigt, da beide Wissenschaften ihren Weg für sich gingen. Der Physiolog, der alle Erscheinungen des organischen Lebens zu berücksichtigen hat, fühlt das Bedüríniss einer Vereinigung um so mehr, weil er 
obne sie nicht fortschreiten kann. Dies ist schon lange von einem unserer vorzüglichen Pflanzenphysiologen gefühlt und ausgesprochen, aber leider nur zu wenig beherzigt worden. Herr Link sagt schon in seinen "Grundlehren der Anatomie und Physiologie der Pllanzen" (p. 5.): „Es ist gut die Grenzen der Wissenschaften scharf zu ziehen, aber es ist auch gut, sie da miteinander zu verknüpfen, wo sie einander Hülfe leisten können." Unsichere Bestimmung der Begriffe muss Verwirrung hervorbringen, zumal wenn sie Urbegriffe wie organiseh and unorganisch betrifft.

So lange als wir einen Organismus von keiner zu niedern Ausbildung vor uns haben, sind wir nicht über seine organische Natur in Zweifel. Anders ist es jedoch, wenn wir den Organismus seinen Elementartheilen nach betrachten, oder einen Organismus der niedrigsten Bildungsstnfe vor uns baben. Hier scheint die scharfe Grenze zwischen Organischem und Unorganisthem aufzuhüren, besonders wenn wir einige niedere Algenformen, die Nullipora etc., ferner gewisse Bestandtheile der höhern Organismen, welche sogar die Chemie darzustellen vermag, betrachten. Hier muss sich jeder fragen: Wo fïngt das Organische an und wo hört es auf? - Diese Frage ist es, welche ich jetzt zu erörtern versuchen will.

Kieselerde, koblensaurer und phosphorsaurer Kalk, Wasser etc. sind Verbindungen, welche Niemand für organisch erklären wird, aber sie kommen als Bestandtheile in organischen Körpern vor. Daraus ergiebt sich, dass ein organischer Körper nicht aus organischer Substanz allein bestehe, sondern dass er auch unorganische Substan:en in sich einschliesse. Da nun aber diese und andere unorganische Verbindungen constant auf bestimmte Organismen angewiesen sind, so ist ihre Gegenwart sogar nothwendig, sei es nun um die organische Substanz durch Anfüllang der Zwischenräume, oder durch Ueberzug der Membranen, Fasern etc. mehr zu befestigen, zu schützen, oder ibre Bestandtheile zur Bildung neuer organischer Materie herzugeben. Jedeufalls ist aber das Unorganische dem Organischen im Organismus untergeordnet, wie dies die Form jener in den Organismen befindlichen Körper bezeugt, welche sich nach der Btructur der organischen Substanz richtet.

Nun kommen aber auch noch Verbindungen in den Orga- 
nismen vor, welche binsichtlich ihrer elementaren Zusammensetzung dieselben Bestandtheile wie dic wirklich organischen Theile des Organismus besitzen. Ich meine biermit die sogenannten organischen Süuren, die Alkaloide, Oele, Harze, Fette, kurz alle diejenigen aus Kohlenstoff, Wasserstoff, Sauerstoff, oder auch aus Stickstoff bestehenden Verbindungen, welche keine organische Structur besitzen. Sollen diese Verbindungen auch als organisch betrachtet werden, weil sie mit der wahren organischen Substanz gleiche.Bestandtheile haben? Sollen sie als organiscle betrachtet werden, weil sie sich im Organismus erzeugten? - Nein! - Denn, wenn wir alles Organische nach seinen Bestandtheilen bestimmen wollten, wie kïmen das kohlensaure, ameisensaure, essigsaure Ammoniak, der Alkohol, Aether etc. dazı, als organische Körper zu gelten? - Und wenn der zweite Grund gelten soll, müssten wir dann die Kieselerde, die chemischen Verbindungen des Kalks etc., die in deo Organismen gefunden werden, nicht auch als organisch belrachten?

Wir sehen daraus, dass man mit der Chemie nicht.ausreicht diese Begrilfe festzustellen. Schlagen wir daher einen andern Weg ein, \&er uns sicherer fübrt.

Alles was rein organisch ist, wirkt nur durch den Tastund Gefühlssinn auf uns und niemals luat es Gesclunack und Geruch. Dicse letztern Sinne sind nur Ragentien für unorganische Verbindungen. Was daher Geschmack und Geruch bat, ist den unorganischen Körpern beizuzählen, selbst wenn es in and an organischen Körpern vorkommt.

Es giebt freilich nur wenige positive Kennzeichen des Organischen, wenn wir es in seiner Elementarform betrachten, oder es auf der niedrigsten Bildungsstuce antreften. In solchen Fïllen kann nur das Microscop entscheiden, aber ein geübter Blick wird sich hier nie tïuschen, es sei denn, dass das $0 r-$ ganische noch keine bestimmte Form, noch keine Structur habe, wie z. B. Schleim, Gallert.

Die organische Substanz ist mehr oder weniger fest, flexibel, nnd besitzt cine eigenthümliche (organische) Slructur, die sich dem Auge in verschiedenartigen, nur ibr zukommenien Gestalten, entweiler als Iemhran, Faser, oder als (hüufig kugeliger) Kern darstellt. Die Membran ist entweder flach ausgebreitet, oder bildet Bläschen, Rühren etc., dic oft wieder ühn- 
liche, oder auch andere Gebilde eiuschliessen. Es geht hieraus hervor, dass der sogenannte Faserstof, ferner Amylon, Fungin, Medullin, Pollenin, und wie die Chemie diese Planzenorgane nennt, keine chemischen, sondern wirkliche organische Verbindungen sind; dagegen alles Tropfbarlüssige, Gasförmige, alles Schmeckende, Riechende, Steinige, Krystallinische, Schmelzhare, Fener-Beständige und Flüchtige, kurz Alles, was die Zellen oder andere hohle Räume in den Organismen erfüllt und mit Wasser, Oelen, Alkohol, Aether etc. ausziehbar ist, gehört nicht za den wahren organischen Gebilden, es sind unorganische, so ungewohnt es uns auch durch den gewöbnlichen Sprachgebrauch in der sogenannten organischen Chemie jetzt scheinen mag. Zwar gehören sie zu den Bestandtheilen des Organismus, aber zu den unorganischen, indirecten, wie der koblensaure und phosphorsanre Kalk, die Kieselerde etc.

Die Chemie kann zur Erkennung der organischen Substanz nur negativ beitragen. Wir wissen, dass eine organische Substanz als solche niemals eine chemische Verbindung eingent, 'wol aber gehen die Bestandtheile organiscier Substanzen chemische Verbindungen ein.

Wenn man von Verbimdungen der Stärke mit dew Jodin, des Faserstoffs mit den Farbestoffen etc. redet, so wird dadurch lkeineswegs jener Ausspruch widerlegt. Bei einer chemischen Verbindung muss auch jeder in der Verbindung befindliche stoff als solcher auch wieder ausgeschieden werden künnen, aber Niemandem ist es gelungen und wird es gelingen, das Stïrmehl als Stärkmeht (nit organischer Structur) von der Jolinstärke zu trennen. Schon wenn es mit blossem Wasser gékocht ist, ist das Stärkmehl kein Stïkmehl melir und wenn Jodin hinyukommt, so verbindet sich nicht das stärkmehl mit jhm, sondern der Kohlenstoff, Wasserstoff und Sauerstoff desselben. Wenn der Faserstofl diese oder jene Farbe leichter annimmt, ist dies darum der chemischen Verwandtschaft zuzuschreiben?

Aus diesen Betrachtungen geht hinreichend hervor, dass die Chemie nie von organischen Verbindungen reden kann, denn, streng genommen, sind alle ihre Verbindangen, die sic darsteilt, unorganisch, weun man auch organische Stoffe dazu verwendet hatte.

Obgleich ich nun hierdurch das Organische und Unorganische so bestimmt, als es mir möglich ist, bezeichnet habe, so hat dennoch die Erkennung der organischeil Substanz be- 
sonders da ihre Schwierigkeiten, wenn sie so nnausgebildet auftritt, dass man oft noch keine Structur an ihr wahrnehmen kann. Sie erscheint in diesem Falle immer sehr weich und sogar flüssig. Diese Beschaffenheit rührt dann von der Anwesenheit einer unorganischen Verbindung her, nämlich des Wassers. Ein solches inuiges Gemenge von organischer Substanz mit Wasser stellt sich nun in der Form von Schleim oder Gelatina dar und ist als solche in vielen Flüssigkeiten der organischen Körper enthalten.

In djesem Schleime können wir nan wohl schon die organische Bildung annehmen, aber die Atome derselben haben sich noch nicht zu einem Körper von organischer Structur vereinigt. Er erscheint uns in dieser Weise als der erste Ausdruck alles Organischen, als der Organismas auf der niedrigsten Stufe. In so fern aber, als auch Körper von organischer Structur sich in den structurlosen Schleim umwandeln, kann er auch im letztern Falle wieder als das lelzte Stadium alles Organischen betrachtet werden, weil durch dieses der Organismus den anorganischen Kräften, dem Chemismus anheimfällt.

Verfolgen wir nun von hier aus die Erscheinungen, welche sich bei der Bildung eines jeden organischen Küruers darstellen, so giebt es keinen einsigen Fall, wo sich neben dem nrganischen Producte zugleich auch ein unorganisches - eine chemische Verbindung - erzeugte. Es ist dies eine Thatsache, die ich durch eine Reihe von zahireichen Beobachtungen gefunden habe. Sie setzt aber auch voraus, dass während der Buldang eines jeden organischen Körpers zugleich zwei Krufte, die organisirende Lebenskraft und die chemische Verwandtschaft thätig sind. Beide sind im organischen Leben stets im Kampfe mit einander begriffen und so lange ein Körper noch organisches Leben besitzt, ist die organisirende Lebenskraft vorherrschend. In diesem Falle kann dieselpe Einfluss auf die Bildung der im organischen Körper sich erzeugenden chemischen Verbindungen ausüben, and daraus allein wird es uns erklärlich; wie im Organismus sich anorganische Verbindungen bilden, welche die Kunst in chemischen Laboratorien uoter gewöhnlichen Verhältnissen nicht immer herzustellen vermag.

Wenn nun die Beobachtungen über den Ernäbrungsprocess 
der Pflanzen es fast ausser $\mathbf{Z}$ weifel setzen, dass die Nahrungsflüssigkeit, welche die Pflanzen aus der Erde aufnehmen, vorzüglich in Wasser aufgelöste Kohlensäure sei, so geht daraus hervor, dass die organisirende Kraft diese anorganischen Gebildo so $z u$ verăndern vermag, dass sie in ihre Elemente zerlegt werden, welche sie im Freiwerden zu organischer Masse verbindet, die rom Organismus assimilirt wird. Daraus folgt aber auch nothwendig, dass sich überhaupl organische Masse aus unorganischen Verbindungen bilden kann, wenn diese nur die Bestandtheile enthatlen, welche sur Zusammenset:ung der oryanischen Masse nöthig sind, und die unorganische Verbindung nicht eine solche ist, welche werstörend auf Organismen wirkt.

Sind daher in einer Flüssigkeit diese Bedingungen gegeben, so können sich auch in ihr organische Gebilde durch Urbildung erzeugen.

Die unumstüsslichen Beweise von Urbildung organischer Materie liefert uns die Entstehung der Hefe und Essigmutter, sie wird selbst den noch Ungläubigen von der Wahrbeit dieser Annahme überzeugen. Aber ich kenne neben dieser noch eine Menge von Thatsachen, von welchen ich in dieser Abbandiung eine Anzahl mittheilen werde. Diese Bildungen werden sämmtlich von ähnlichen Erscheinungen begleitet, welche wir bei der Bildung der Hefe und Essigmutter kennen lernten. Da die Bildang der Hefe und Essigmutter schon bekannter ist, so will ich mit díeser den Anfang machen und an ihnen dic Theorie entwickeln, die dann auf alle Erscheinungen des organischen Lebens, besonders der Pllanzen, Anwendung findet.

Wird eine geeignete Flüssigkeit der geistigen Gährung unterworfen, so bildet sich neben der Hefe zugleich Kohlensäure und Alkohol, also neben der organischen zwei unorganische Verbindungen. Die Bildung dieser drei versehiedenen Producte geht so lange fort, bis noch etwas von den gährungsfaihigen Bestandtheilen in der Flüssigkeit entbalten ist. Ist die bierbei erhaltene geistige Flüssigkeit mit hinreichendem Wasser verdünt, so geht sie bei angemessener Temperatur und beim $\mathrm{Zu}$ tritt von atmospbärischer Luft in die saure Gährung über, es entsteht wieder ein organisches und ein unorganisches Gebilde, Essigmulter und Essig. 
Die Erfahrung lehrt, dass mit der Menge der hierbei gebildeten anorganischen Producte auch die der organischen im Verhailtniss steht, und dass die Bildung der letztern auch nothwendig zur Bildung der erstern ist.

So betrachtet, muss uns jeder Gährungsprocess jetzt anders erscheinen, als er bisher in der Chemie betrachtet wurde. Es ist bekannt, dass die Chemie die weinige Gährung durch die Einwirkung des sogenannten Klebers auf das Amylon und den Zucker erklärt; ich muss aufrichtig gestehen, dass ich mir Jurch diese Erklärung den Vorgang nicbt deutlich machen kann. Ob Andere glücklicher darin sind, möchte ich bezweifela. Sicher hängt aber der ganze Process bei der geistigen Gührung: von der Bildung der Hefe, und bei der sauren von der Bíldung: der Essigmutter ab $*$ ). Diese Organismen bilden sich unter allen entsprechenden Verhältnissen, wenn die Elemente zu ihrer Bildung vorbanden sind. Ist aber der Anfang zu ibrer Bildung gemacht, so ist auch mit ihrem Erscheinen zugleich die Bedingung gegeben, sich fortzupflanzen, d. i. sich zu vervielfältigen. Mit dem grössern Anwuchse dieser Organismen vermehrt sich auch der Vervielfültigungstrieb und mit diesen nimmt zugleich die 'Einwirkung auf die vorhandene Flüssigkeit zu, deren übrige Bestandtheile, welche nicht in die organische Bildung mit eingehen, zu anorganischen Producten, Kohlensïure, Alkohol, oder Essigsäure sich vereinigen. Das der Vervielfïligungstrieb jener Organismen wirklich es ist, weluher hauptsächlich jene Zersetzung der Flüssigkeit zuerst anreğt, beweist nicht nur, dass durch Hinzufügen der Hefe und Essigmutter die geistige und saure Gahrang schneller und sicherer eingeleitet werden können, sondern auch, dass ein Stillstand der Gährung eintritt, wenn Hefe und Essigmutter aus den giihrenden Flüssigkeiten vollkommen entfernt werden. Auch erklärt sich hierdurch die Thatsache, dass ausgetrocknete Hefe nicht zur Einleitung der geistigen Gahrung tauglich ist, weil sie in diesem Zustande einem todten, vertrockucten Organismen gleicht. Da aber die Hefe sich auch durch Urbildung erzeugt, so kann

*) Diese Erklärung, welche ich schon 1834 niederschrieb, aber im Minniscripte liegen liesú, schliesst sich im Wesentlichen, wenn auch uit einigen Modificationen an die des Herrn s c h wan u al. 
ihre Billung nach dem Zerstören der abgestorbenen Hefe wieder aus den Bestandtheilen derselben veranlasst werden.

Vergleichen wir nun diese Erscheinangen mit denen, welche sich bei dem Lebensprocesse der höhern Pllanzen darbieten, so finden wir in der That eine aufallende Uebereinstimmung mit ihnen.

Wenn der Embryo sich entwickeln soll, so bedarf er der Luft, des Wassers und einer angemessenen Temperatur, Bedingungen, welche ebenfalls die Gührung erfordert. Entwickelt sich der Embryo, so bemerlien wir, dass gleichzeitig das Amylon des Eiweisskörpers in Zucker- und dieser in Kohlensäure und Ussigsäure *) verwandelt wird. Diese Verïnderung des Zuckers in Kohlensäure ist nöthig, weil diese das eigentliche Nahrungsmittel des Embryo, wie überhaupt der ganzen Pflanze ist.

Ich werde jetzt eine Reihe von Beobachtungen noch mittheilen, die das eben Gesagte noch in vielen einzelnen Fällen bestiitigen sollen.

I. Pflanzengebilde im Quittenschleim.

(Fig. IX.)

Wenn Quittenschleim im Sommer einige Tage alt wird, so bildet sich nach und nach eine rusammenhängende, flockigschleimige Substanz, während die übrige Flüssiglieit an Mucositït verliert und dünnllüssiger wird. Lacmuspapier wird in diesem Falle von der noch übrigen Flüssigkeit stets gerüthet, und weun man jene Schleinflocken unter Icm Microscope betrachtet, so sielt man, dass sie aus aggregirten, licinen, schleimigen Kügelchen bestchen, wie sie die Abbildung zeigt.

II. Pflanzengetilde ip destillirten Wässern.

Es ist eine besonders den Pharmaceuten längst bekannte Thatsache, dass destilirte Wässer nach längerem Aufbewabren ihren eigenthümlichen Geruch verlieren und statt dessen einen andern, moderigen Geruch bekommen. Mehrere solche Wässer, welche ich nach ihrem Verderben untersuclite, reagirten stets

ij Vergl. Becquerel in Annal. de Chimie et de Pbysique. Mars. 1832. 
sauer, wie z. B. aqua rubi idael, aqua valerianae und aqua sambuci: Es findet sich aber in diesem Falle auch stets, dass schleimige Flocken in dem verdorbenen Wasser schweben, die sich oft auch an die Wände, oder auf den Boden des Gefässes lagern. Diese Schleimflocken sind vegetabilische Gebilde, von welchen besonders Herr Dr. Biasoletto eine ziemliche Anzabl in seinem schon oben erwähnten Buche beschrieben und abgebildet hat. Agardh beschreibt in seinem Systema algarum ebenfalls mehrere derartige Bildungen, z. B. in der aqua salviae, aqua rosaram. Ich habe ebenfalls schon früher diese Gebilde in einem Aufsatze "Beitrag zur Kenntniss über die Entstehung und Metamorphose der niedern vegetabilischen Organismen etc.", welcher in der Linnaea 1833. Heft III. abgedruckt wurde, erwähnt, ond damals schon darauf aufmerksam gemacht, dass die schleimig gewordenen destillirten Wässer stets ihren eigentlümlichen Geruch nach atherischem Oele mehr oder weniger verlieren. Da ich mehrere Formen derselben untersucht habe, die noch nicht von andern näher untersucht und beschrieben worden sind, so theile ich die Resultate darüber mit.

a) Pflanzengebilde in der aqua calami.

(Fig. X.)

Dieses Vegetabil bildet eine sehr schlcimige und schlüpferige Haut, welche den Boden eines Glasgefisses, in welchem das Calmuswasser aufbewahrt wurde, überzog. Unter dem Microscope zeigten sich grössere Kugeln (4mal grösser, als die Helekügelchen), welche unter sehr kleinen Schleimlïugelchen zerstreut lagen. Diese grössern Kugeln zeigten ebenfalls einen sehr feinkörnigen Inbalt und waren meist zu mebreren aneinander gereiht, wie die Perlen an einer Schnur. Die aqua calami reagirte sauer.

\section{b) Pflanzengebilde in aqua valerianae.}

\section{(Fig. XI.)}

Dieses batte sich ebenfalls auf dem Boden des Gefässes, in welchem das Baldrianwasser anfbewabrt wurde, gelagert. Beim Umschülteln schwebte es als unregelmässig zerrissene Flocken im Wasser herum, welche, unter dem Microscope be- 
trachtet, aus einer Menge Kügelchen bestanden, die etwas kleiner, als die Hefekügelchen waren, sich aber alle mehr oder weniger regelmässig aneinander gereihet hatten. Hierdurch wurden perischnurförmige Fäden gebildet, die sich meist parallel nebeneinander gelagert batten. Manche dieser Fäden verästelten sich auch und trugen an ihren Enden traubig aggregirte Kügelchen. Die aqua valerianae reagirte ebenfalls sauer.

c) Pflanzengebilde in der aqua rabi idaei.

(Fig. XII.)

Es stellt kleine zerrissene Flocken dar, die aus ungegllederten und sparrig verästelten Fäden bestehen, welche hie unil da blasige Anscbwellangen tragen. Diese Anschwellangen sind 4mal grösser, als die Hefekügelchen. Sie sind jedenfalls den Fructificationsorganen mancher Algen (z. B. der Vaucheria) analog. Das Wasser reagirte sauer.

d) Pflanzengebilde in aqua lauro-cerast.

(Fig. XIII.)

Dieses wurde mir von einem jungen Pharmaceuten, Herrn Beez, vor einigen Jahren mitgetheilt. Es bestand aus verworrenen sehr feinen Fäden, an welchen ich nur sehr andeutliche Gfiederung wahrnehmen konnte, die meisten erschienen völlig ungegliedert und nur hie und da waren cinige, zu kleinen Schnüren aneinander gereihete Kügelchen bemerkbar.

e) Pflanzengebilde in der aqua salviae.

(Fig. XIV.)

Es stellt eine sehr schleimige und schlüpferige Substan\% dar, welche unter dem Microscope aus verästelten Fäden bestehend erscheint. Diese Fïden zeigen in gewissen Entfernun gen hyaline Puncte. Aussen an den Fäden finden sich noch sowobl traubig gruppirte, als anch einzelne kleinere hyaline Kügelchen (a) und ausserdem sieht man auch noch grüssere opake Anschwellungen (b). Auch dieses Wasser reagirte, jedoch nur sebr schwach, sauer. 


\section{I. Pflangengebilde in fetten Oelen.}

\section{(Fig. XV.)}

Es ist bekannt, dass sich in fetten Oelen nach längerem Aufbewahren eine schleimige Substanz absondert, die sich auf dem Boden des Gefässes sammelt. Zugleich bemerkt man auch, dass die Oele rarkig werden und saver reagiren.

Dieser schleimige Absat\% ist ebenfalls eine vegetabilische Bildung. Ich habe den Schleim, welcher sich im Mohnül absetzt, untersucht, und gefunden, dass er aus dicht verschlungenen, istigen Faiden besteht. Der Hauptstamm der Fäden ist stärker, als die Aeste, dabei gegliedert; die Glieder 3-6mal länger, als ihr Querdurchmesser. Am Ende verlängert sich def Hauptstamm des. Fadens in einen dünnern, ungegliederten Falen, und ebenso erscheinen nuch die Nebenäste. : Aussen tragen die Fïllen an; manchen Stellen kugelige, durchsichtige Sporen, die ich theils. einzeln (a), theils zu Gruppen vereinigt (b) fand. Diese Sporen bilden wieder den ersten Anfang junger Pfl̈̈nzchen, wie die Fig. c zeigt.

1V. Pflanzengebilde in einer Auflösung von brenzlichem, bernsteinsaurem Ammoniak (Liquor ammonii suctinici der Preuss. Pharmacopöe].

(Fig. XVI.)

Dieses bildet eine fiulige, flockige Schlcimmasse, welche sus melirmals wiederbolt verästelten Fïden besteht, die rom Hauptstamme an, bis in die äussersten feinsten Eoden geglielert èrschernen. Die Glieder des Hauptstammes sind 6-12mal länger, als ihr Querdurchmesser und enthaiten kleine opake Kügelchen, welche wie kleine Gürtel im Faden erscheinen. Die Glieder der Aeste, welche ebenfalls diese opaken Gürtelstreifen zeigen, werden bach den Enden zu kürzer.

V. Pflauzengebilde in spirituosen Flüssigkeiten. (Fig. XVII.)

Herr Apotheker Hornung in Aschersleben theilte mir ein vegetabilisches Gebilde mit, welches sich in rectifrcirtem Weingeist und im Kampherspiritus vorgefunden hatte. Ba es mir. nicht wahrscheinlich scheint, dass dieses Gebilde. sich erst in diesen Flüssigkeiten erreugt habe, so vermuthe ich, dass sich 
dieses Vegetabil in dem hölzernen Gefässe, in welchem der Weingeist aufbewahrt worde, gebildet habe, bevor es mit Weingeist gefüllt wurde. Da ich jedoch hiervon eben so wenig Gewissheit habe, als yon dem wirklichen Erreugen dieses Vegetabils in Weingeist selbst; so mache ich durch diese Mittheilung darauf aufmerksam und füge die Beschreibung dieses Vegetabils hinzt. Die Füden sind sehr dünn und ästig, die Aeste oft ganz Kurz, spityig und sparrig abstehend. Die Gliederung dur Fäden ist nur bei etwas matter Beleuchtung $z u$ bemerken.; doch scheint es keine wirkliche Articulation zu sein, sondèn nar eives scheinbàre, hervorğebracht durch deutlich unterschiedene Kügelchen, die sich der Länge nach in dem Innern des Fajens vorfinden, wie aus der Abbildung zu ersehen ist. bós Vegetahil, welches ich Fig. XVir. abgebildet hábe, ist aus rectificirtem Weingeiste. Mit diesem stimmt dasjenige aus dem Kampherspiritus vollkommen überein. Der letztere war nümlich mit demsetben-Weingeist bereitet worden, in welchem - sich jenes Vegetahil varfand.

Vto Pflanzengebtrie in phos horsāure.

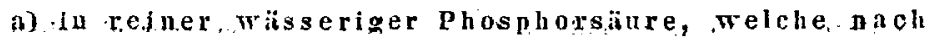
Vorschrift der Preuss. Pharmacopöe durch oxydation des Phosphors mattelst salpetersitue erhalten war.

(Fig. XVIII. a.)

Ich. verdanke die Mittheilung: dieses productes ebenfalls dem Herrn Beez. Es hat viele Aehnlichkeit mit einem Sporotrichum. Die Fäden sind ungegliedert, gleich dick, ästig; die Aeste divergirend, gerade and steif. Aússen án den'Fïden sitzen sowohl kugelige als In̈ngliche Sporen.

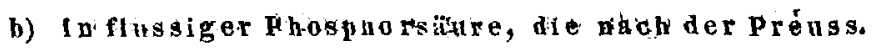
Plearmacopoe alos kochen bereitet war.

(Fig. XVIH: b.]

Bildet eme-faserige Schleimmasse. Die Fäden sind etwas wenges starker, als bel voriger Form, auch deutlich giggliéaerı, die Glieđer meist $\mathbf{3}-\mathbf{4}$ mal lānger, àls ibr Dúrchmesser. Vie Aeste sind abstehend und meist gehogen, nicht steif. Hie und da sitzen an den Fatden einige runde sporen. 
404 Kü tzing, üb. Hefe und Essigmutter.

VIf. Pflanzengebilde in elner verdinnten Anflögng von Brechweinstein.

(Fig. XIX.)

Es bildet eine selir zarte, flockige und lockere Schleimmasse, bestehend ans ästigen, gleichstarken Fäden, die anfangs ganz ungegliedert sind, Jeren Enden sich aber in perlschnurnrtige Glieder auflösen. Es schliesst sich diese interessante Form an die Pilzgattung Oidium an. - Die Brechweiusteinauflösung verïnderte sich jedesmal aup die Weise, dass sich Antimonoxyd ausschied. ${ }^{-1}$ Aus diesen Grunde ist es daher unzweckmüssig, wenn in Apotheken Solutio tartari stibiali vorräthig gehalten wird.

VIII. Delanzengebilde, welche in wässeriger Polychromlösung entstanden.

a) in reiner, wässeriger Polychrombsung. (Fig. XX. [jünger] u. Fig. XXI. [älter]).

Dieses erschien als eine olivenfarbige Schleimmasse, welche in sehr lockern, zarten und wolkigen Flocken die Auflōsung trübte.: Unter dem Microscope zeigten sich dichotomisch verzweigte und deutlich gegliederte Fanden, deren Aeste bald aufrecht, lang und schlank (Fig. XX), bald sparrig abstehend und kürzer waren (Fig. XXI.), letztere waren aussen mit kleinen Sporenbäufchen (a) besetzt. Der Unterschied zwischen beiden Formen liegt in dem verschiedenen Alter, Fig. XXI. ist nầmlich die altère Form; wejche daher auch fractificirend erscheint.

b) in wässeriger Polychramlösang, die mit Cblorgald versetzt war.

(Fig. XXII. [jünger]. Fig. XXIII. [ä]ter]).

Dieses Vegetabid überzog den Boden des Glases: als eine dunkele, braunviolette, schlüpferige und faserige Schleimrnasse. Unter dem Microscope zeigte es sich (im jüngern Stadium, Fig. XXII.) als dicht verworrene, meist. dichotomisch verästelte Fäden, die zum Theil, namentlich am Hauptstamme, gegliedert erschienen; die Glieder des Hauptstammes zeigten noch eine breite Querzone. Die Aeste und schwächeren, Făden erschieuen mit inwendigen, in gewissen Absätzen gereibeten sehr 
hyalinen und daher nur schwierig siehtharen Kügelchen. Zwischen diesem Fadengewebe lagerten Aggregate von grössern Kügelchen, ohngefähr $11 \%$ bis $2 \mathrm{mal}$ grösser, als die Hefekügelchen (a). Diese Kügelchen erschienen durch ihre dicke schleimige Hülle mit einem hyalinen Rande und waren auch öfter perlschnurartig aneinander gereilht (b. c. d.). Soll ich meine Ansicht über diese Kügelchen aussprechen, so möchte ich sie für den Anfang des eben beschriebenen Vegetabils betrachten, wozu ich mich durch die Beobachtung über die Fntwickelung der im nächsten Abscbnitte $c$ folgenden Bildung veranlasst finde.

Noch muss ich erwähnen, dass ich eine äusserst kleine, nur schwierig erkennbare Vegetation aur den Fïden der eben beschriebenen Bildung beobachtete, die ich für einen kleinen Schmarotzer halte. Die Figuren $g$ stellen dieses kleine Vegetabil dar. Es bildet kleine, längliche, spindel - oder auch keulenförmige Körperchen, die auf äusserst Peinen, nur darch die Bewegung der Flüssigkeit sichtbaren stielchen sitzen. Diese Körperchen sind oft fächerförming oder auch doldenförmig gruppirl.

Im ältern Stadium ist bei dem oben beschriebenen Vegetabil die Gliederung der Fäden völlig entwickelt (Fig. XXIII.), so dass sie bis in die aussersten Spitzen Uer feinsten Aeste zu verfolgen ist. Die oben erwähnte Gruppirung der Kügelchen ist verschwunden, wabrscheinlich weil sie in ordentliche Fäden ausgewachsen sind.

In der Flüssigkeit hatte sich regulinisches Goldpulver niedergeschlagen und auf der vegetabilischen Substanz abgesetzt.

c) in wisseriger Palychromlösung, die mit Chlorgald und Zinnchlorïr versetzt war.

(Fig. XXIV.)

Diese Substanz bildete eine braunviolette, schleimige, flokkige Masse, welche aus verschiedenartigen Füden, vermischt mit einzelnen Kügelchen, den Anfang des Vegetabils bezeichnend, bestand. Das fädige Gewebe bestand theils in sehr dünnen, meist ungegliederten, theils stärkern, torulusen oder perlschnurartigen Fïden. Bei genauer Untersucbung zeigte es sich, dass die dünnern, ungegliederten Fäden theils die Aeste, theils die Euden der stärkern Fäden waren. Die Figuren $a, b, b^{\prime}$, 
c, $d$ und e, zeigen auf das Deutlichste, wie die Entwickelung: der Fäden aus den kugelförmigen Uranfängen stattfindet.

Es hatte sich auch hier das Gold als Peines regulinisches Rulver, mit etwas Zinnoxyd vermischt, aus der Flüssigkeit niedergeschlagen.

d) in wässeriger Polychromlösung, die mit Zinnchlorid versetzt war.

(Fig. XXV.)

Aut dem Boden des Gefïsses hatte sich ein Niederschlag: von Zinnoxyd gebildet, der als Unterlage der unmittelbar darauf gewachsenen Bildung diente. Sie stellte sich als eine schlüpfrige, gelbe Schleimmasse dar, die aus stärkern und sohwächern, gegliederten und ästigen Fäden bestand. Aussen an den Fäden sassen hie und da trauhig grappirte, sehr kleine kugelige Sporen (a), aus welchen ich selbst wieder jüngere Fäden sich entwickeln sah (b). Diese letztern waren so äusserst fein, dass ich keine Structur an ihnen wahrnehwen konnte.

e) in wässeriger Polychromlöstign, die mit Brecbweinstein versetzt war.

(Fig. XXV1.)

Diese Bildung zeigte sich als ein selar weicher, nur wenig zusammenhängender, fast flüssiger Schleim, welcher aus sehr kleinen, kurzen, einfachen perlscburartigen Fäden bestand, die aus äusserst kleinen Kügelchen (J̆mal kleiner als die Hefekügelchen) zusammengesetzt waren. Mit diesem hatte ein anderes Product ganz gleiche Bildung, welches sich in einer andern wãsserigen Polychromlösung, die mit Chronsäure verset $\iota$ war, erzeugt hatte.

f) in wässeriger Polychromlösung, die mit schwefelammonium versetzt war.

(Fig. XXVII.)

Diese vegetabilische Bildung stellte ein dunkelbranes faseriges, wenig schleimiges Gewebe dar, welches aus ziemlich starken, dichotomisch verästelten Fäden bestand. Diese starken Fäden zeigten zum Theil keine, oder nur undeutliche Gliederung waren durchaus opak und braun gefürbt, und aus ihnen 
gingen wieder äusserst feine, verworrene, ästige, durchisichtige und farblose Fäden aus. Ausserdem bemerkte ich unter den gliederlosen, stärkern und opaken Füden, ebenfalls einmal einen opaken Faden (a), der aus kugeligen, gebräunten Gliedern bestand; auch fanden sich öfters ähnlich gegliederte, kurze Aeste an den stärkern, ungegliederten Fäden (b).

g) in wässeriger Polychromlösng, die mit schwerelsaurem Kupferoxyd versetzt war.

(Fig. XXVIII.)

Diese Vegretation bildete eine sehr schlcimige, schlupterige Masse, die fest anf dem Boden des Gefitsses sass. Sie bestand aus sehr dünnen, meist dichotomisch veristelten Fïlen, die inwendig, in gewissen Entfernungen, kleine, hyaline Kügelchen cnthielten. Aussen enthielten sie Aggregate von läuglichen Sparen (c). Dieses Gcbilde erzengte sich aus sehr kleinen Kügelchen (a), wozu die Fig. b. den Beleg liefert.

An diese Mittheilungen erlaube ich mir nun noch einige Thatsachen anzuschliessen, die zwar den Chemiker und Pliarmaceuten schon bekannt sind, aber noch nicht in eine allgemeine Betrachtung gezogen wurden.

Wenn man nach der alten Scheel'schen Methode Gallussëure darstellen will, so lïsst man die Galläpfelinfusion schimmeln. Es bilden sich dann braune Krystalle von Gallussäurc darin. Wer findet hicrbei nicht sogleich dieselbea Erscheinungen wieder, die sich uns bei der Wein- and Lssiggahrung darbieten? - Schinmel (ein vegetabilisches Gebilde, der Hefe, Essigmutler und den oben beschriebenen Vegelationen analog) bildet sich jedesnual dabei und seine Bildung ist hier von der Bidung der Gallussïure unzertrennlich.

In Journal für practische Chesnie 1834. I. p. 475. habe ich nachgewiesen, dass die l'Tübung der wässerigen Rhabarbertinctur der Apotheken ebenfalls durch das Ausscheiden eines vegetabilischen Gebilies veranlasst wird. Ich erwibnte schou damals, dass die Verïnderung der Tinctur (besonders auch in Minsicht auf Farbe) mit dem Ausscheilen jener vegetabilischen Kügelchen in innigen, unzertrennlichen Zusammenhange steke. Dass die Tinctur zuletzt ihre dunkele braune farbe verliert und immer heller wird, liasst vermuthen, dass sich neben den 
vegetabilischen Schleimkügelchen eine Säure bildet, die das kobleusaure Kall abstumpft; daher das Verschwinden und Verändern der braunen Farbe. Ich babe in letzter Zeit diese Tinctur nicht wieder untersucbt und es wäre zu wünschen, dass ein geübterer Chemiker die chemische Veränderung derselben, mit Bezugnahme auf das vegetabilische Gebilde einmal genauer untersuchte.

Fassen wir nun alle eben erwähnten Erscheinungen, die sich im ganwen organischen Leben wiederholen und parallelisiren ${ }^{*}$ ], zusammen, so muss uns der Begriff von Gührung jetzt ganz anders erscheinen, als er uns bisher erschien.

Die Gährung ist ein Kampf zwischen organischem und unorganischem Leben (Chemismus), der so lange dauert, bis beide sich ausgeglichen baben. Danı, nachdem sich die dabei gebildeten organischen und unorganischen Producte rein ausgeschieden und von einander getrennt laben, tritt Ruhe eis. In so fern nan Gäbrung gleichbedeutend ist mit einer gegenseitigen Wirkung sich erzeugender, organischer und unorganischer Gebilde auf die Bestandtheile einer gegebenen Flüssigkeit, die in Bezug auf das organische Product als Nahrungsmittel betrachtet werden kann, so ist sie auch nothwendig gleichbedeutend mit jedem organischen Lebensprocess. Daher organisches Leben $=$ Gährung.

Jene Processe dagegen, welche die Essigbildung aus Alkohol mittelst Platinmohr, oder auf andere, diesem ähnliche Weise einleiten, kōnnen nicht mit der Gährung verglichen werden, sie sind rein chemische Processe, während die Gährung ein organisch-chemischer Process, wie der Lebensprocess eines jeden organischen Körpers, ist.

Obgleich es in letzterer Zeit mehr als früher geschab, dass man bei chemischen Untersuchungen organischer Producte die Organisation derselben berücksichtigte, so geben diese Untersuohungen dennach von Neuem den Beweiss, dass die so-

*) Ich erinuere hierbei daran, dass in jedem organischen Kürper, wo sich organischer stoff bildet, der vom Organismus assimilirt wird, sich auch jedtsmal ein oder mehrere unorganische Producte erzeugen, die theils Im Organismus verbleiben (Oele, Fette, Harze), theils auggeftihrt werden (Urin, Milch eto.). 
genannte organische Chemie diesen Gesichtspunct nicht genug Pesthalten kann. Zugleich wird man aber auch immer mehr das Bedürfniss fühlen, dass man die Ausdrücke organisch and unorganisch, wie ich schon oben erwähnte, immer genauer von einander scheide, und namentlich den Begriff organisch in der Chemie in so weit beschränke, als es durchaus nöthig ist, am Begriffsverwirrungen zu verhüten.

Sollte ich hierdurch den ersten Anlass dazn gegeben haben, so bin ich durch das Bewusstsein, der Wissengschaft genüt»t zu haben, hinlänglich belobnt, und verzichte gern aup das Prioritätsrecbt einer Entdeckung, die doch durch das tägliche Fortschreiten der Naturwissenschaften endlich gemacht werden musste.

Ob nun diese in den Flüssigkeiten sich bildenden Vegetabilien als Algen oder als Pilze gelten sollen, überlasse ich der Ansicht eines jeden Einzelnen. Die Mycologen betrachten sie als Pilze, die Algologen als Algen, und beide Theile baben in ihrer Art Recht. Am schwierigsten aber ist die Beantwortung der Frage: ob diese Gebilde in genera und species unterschieden werden können? - Ich habe diese Unterscheidung einmal in einer Zeit versucht, wo ich nur wenige dieser Formen erst antersucht und beobachtet hatte; jetzt bat mich die ungeheure Mannigfaltigkeit der Formen davon zurückgeschreckt. Vielleicht gehe ioh einmal späterhin wieder an diese Arbeit; obgleich ich im Voraus überzeugt bin, dass sich wenig Gewinn für die Wissenschaft hoffen lïsst, seitdem durch diese Entdekkungen die Beziebungen gefunden worden, in welchen diese Gebjlde zur ganzen übrigen Natur stehen.

II.

Ueber feste Verbindungen des Zuckers mit den Alkalien und Melalloxyden.

Von

E. H $\mathrm{D} \times \mathrm{T}$ ○ $\mathrm{N}$.

(Annal. of philos. Ang. 1837.)

Wenn Kalk in eine Zuckerauflösung gebracht wird, so löst er sich in betrâchtlicher Menge auf. Nach U re in der 
Tab.II.

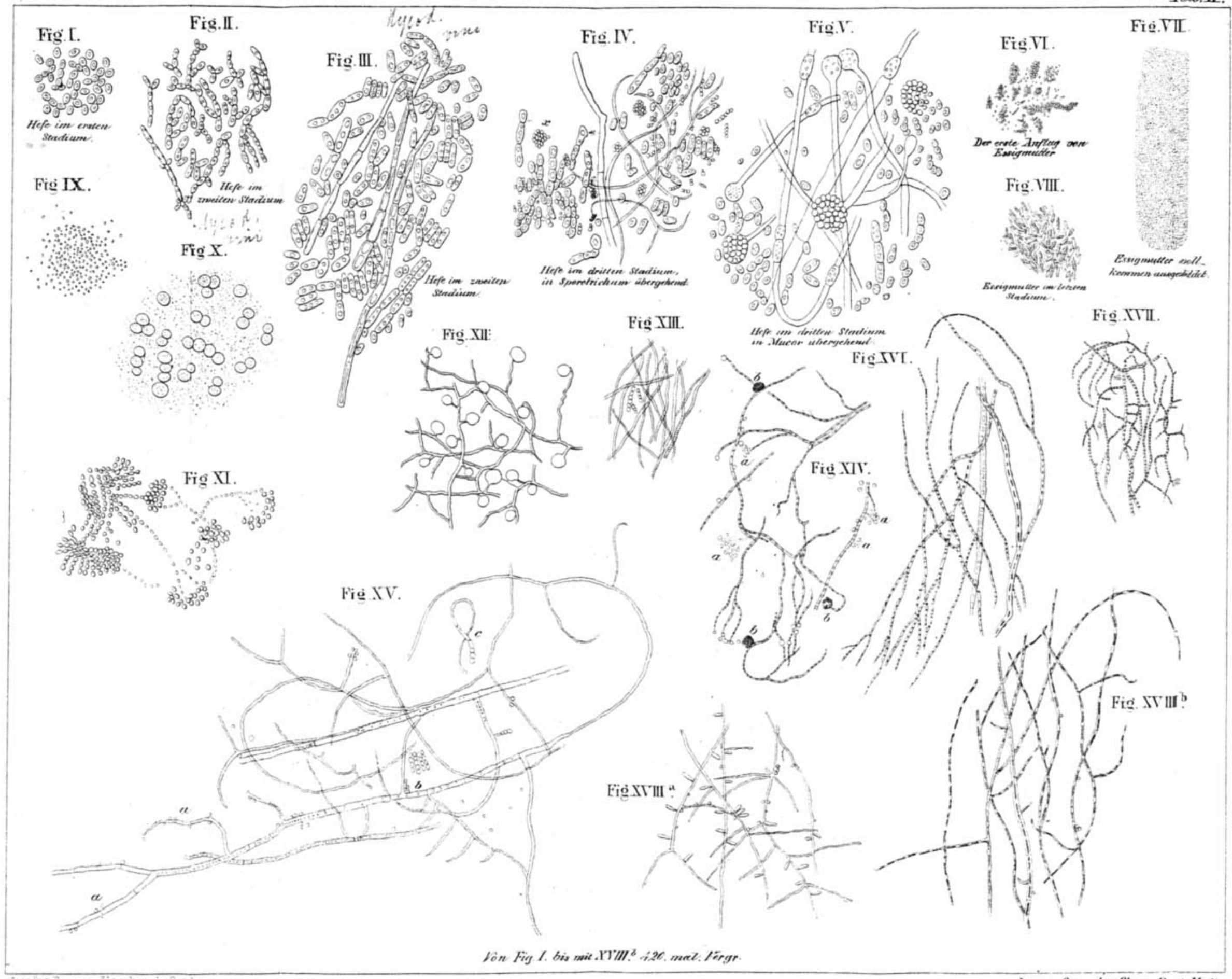


Tab.III.

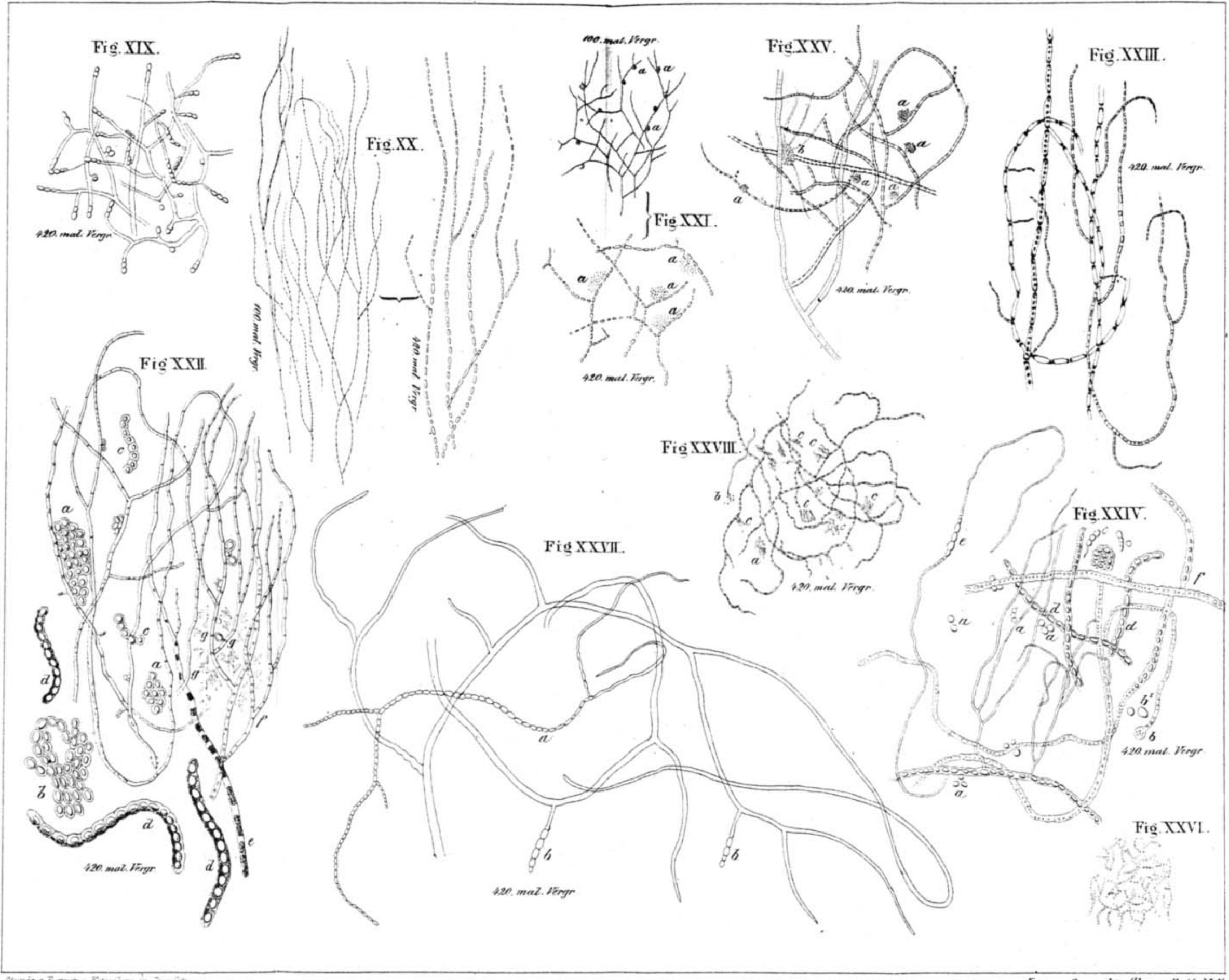

